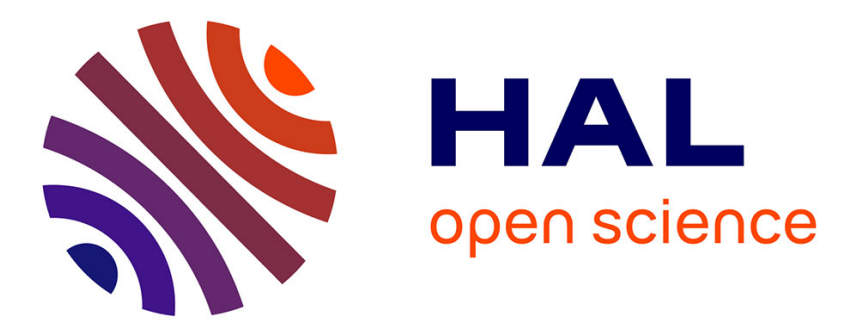

\title{
Ressenti émotionnel positif dans une tâche expérimentale chez l'enfant
}

I. Giannopulu, I. Sagot

\section{To cite this version:}

I. Giannopulu, I. Sagot. Ressenti émotionnel positif dans une tâche expérimentale chez l'enfant. Annales Médico-Psychologiques, Revue Psychiatrique, 2010, 168 (10), pp.740. 10.1016/j.amp.2009.10.014 . hal-00698837

\section{HAL Id: hal-00698837 \\ https://hal.science/hal-00698837}

Submitted on 18 May 2012

HAL is a multi-disciplinary open access archive for the deposit and dissemination of scientific research documents, whether they are published or not. The documents may come from teaching and research institutions in France or abroad, or from public or private research centers.
L'archive ouverte pluridisciplinaire HAL, est destinée au dépôt et à la diffusion de documents scientifiques de niveau recherche, publiés ou non, émanant des établissements d'enseignement et de recherche français ou étrangers, des laboratoires publics ou privés. 


\section{Accepted Manuscript}

Title: Ressenti émotionnel positif dans une tâche expérimentale chez l'enfant

Authors: I. Giannopulu, I. Sagot

PII: $\quad$ S0003-4487(10)00217-9

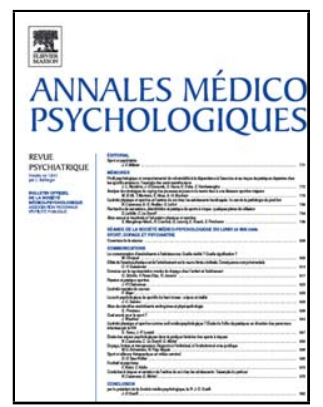

DOI: $\quad$ doi:10.1016/j.amp.2009.10.014

Reference: $\quad$ AMEPSY 1191

To appear in: $\quad$ Annales Médico-Psychologiques

Received date: $\quad$ 7-7-2009

Accepted date: $\quad$ 26-10-2009

Please cite this article as: Giannopulu I, Sagot I, Ressenti émotionnel positif dans une tâche expérimentale chez l'enfant, Annales medio-psychologiques (2010), doi:10.1016/j.amp.2009.10.014

This is a PDF file of an unedited manuscript that has been accepted for publication. As a service to our customers we are providing this early version of the manuscript. The manuscript will undergo copyediting, typesetting, and review of the resulting proof before it is published in its final form. Please note that during the production process errors may be discovered which could affect the content, and all legal disclaimers that apply to the journal pertain. 


\title{
Mémoire
}

\section{Ressenti émotionnel positif dans une tâche expérimentale chez l'enfant} Positive emotion in the course of an experimental task in children

\author{
I. Giannopulu $^{\text {a,b }}$, I. Sagot ${ }^{\text {a }}$ \\ ${ }^{a}$ École des Psychologues Praticiens, 23, rue de Montparnasse, 75006 Paris, France \\ ${ }^{b}$ CNRS FRE 3154, Unité de physiopathologie de la vision et de la motricité binoculaire \\ interaction visuoposturales : ontogenèse et vieillissement, Hôpital Européen G. Pompidou, \\ 20, rue Leblanc, 75015 Paris, France
}

Auteur correspondant : Irini Giannopulu

Tél. : 0142963316

Adresse email : irinigianno@gmail.com

Texte reçu le 7 juillet 2009, accepté le 26 octobre 2010

\section{Résumé}

L'objectif de cette étude est d'analyser si les enfants souffrant de dysharmonie psychotique ressentent comme les enfants «tout venant» les émotions positives. Deux groupes, l'un clinique et l'autre contrôle, comprenant chacun 11 sujets âgés de 8 ans, ont été constitués. Les enfants ont été confrontés à des histoires mettant en scène des émotions positives telles que la «joie », l'«amour » et la «fierté ». Il leur a été demandé de prendre une décision pour terminer chaque histoire et d'évaluer leur propre ressenti. Notre recherche montre, pour la première fois expérimentalement, que les enfants présentant une dysharmonie psychotique ressentent au même titre que les enfants «tout venant» les émotions positives étudiées. Elle suggère également que le ressenti de la «joie» enrayerait l'émergence des représentations associées au vécu de cette émotion chez l'enfant présentant une dysharmonie psychotique. 
Mots clés : Développement ; Dysharmonie psychotique ; Émotion positive ; Prise de décision

\begin{abstract}
The aim of this study is to analyze if the children suffering from psychotic disharmony feel as typical children positive emotion. Two groups, a clinical and a control, including each 11 children 8-year-old were shaped. The children were confronted with stories staging positive emotion such as the "enjoyment", the "love" and the "pride". It was asked them to make a decision in order to complete each story and to estimate their own emotion. The study shows, for the first time experimentally, that the children presenting a psychotic disharmony feel in the same way that typical children the studied positive emotions. It also suggests that the emotion of the "enjoyment" would check the emergence of the representations associated to a previous experience of this emotion at the child presenting a psychotic disharmony.
\end{abstract}

Keywords: Decision making process; Development; Positive emotion; Psychotic disharmony

\title{
1. Introduction
}

Les émotions permettant la création des représentations internes complexes apparaissent très précocement dans le développement humain [32]. Pour le nouveau-né, l'expression faciale, les gestes et la tonalité de la voix sont les premiers moyens de communication avec le monde externe [6,24]. Dès le quatrième mois, l'enfant discriminerait aisément certaines émotions à valence négative comme la peur, la colère, la tristesse, et à valence positive comme la joie [16,31]. Différents types de sourires correspondraient à différents degrés d'intensité de joie [26]. Au-delà de huit mois, l'enfant apprendrait à mettre en relation l'expression faciale, les mots et le contexte, et à mentaliser des séquences typiques de chaque émotion de base [2,3]. La capacité de nommer l'expression émotionnelle, quelle que soit sa valence, surviendrait entre trois et dix ans [31]. La capacité à comprendre et partager les émotions des autres, ainsi qu'à exprimer ses propres états émotionnels, dépend de la maturation cérébrale [32].

Des travaux neuro-anatomiques relatent l'existence des multiples circuits neuronaux qui sous-tendent l'aptitude à reconnaître et comprendre les états émotionnels de l'autre [10,22]. L'importance des connexions cortico-sous-corticales entre le système limbique, les noyaux amygdaliens, l'insula et les régions temporales supérieures et frontales inférieures 
pour la création des représentations associées au ressenti des émotions positives a été souligné récemment [8]. Par ailleurs, il a été montré que les lobes temporal inférieur et supérieur traitant respectivement des informations visuelles [7,28] et auditives [1,2] de plaisir ou de déplaisir associées aux situations externes [12,21], envoient leurs afférences vers le cortex préfrontal [30]. Les émotions positives impliquées dans le guidage de l'action future ou encore la flexibilité des choix nous permettraient de prendre des décisions en faisant intervenir le cortex préfrontal [5]. En d'autres termes, le ressenti des émotions et la prise de décision nécessitent l'intégration des informations multimodales au sein du système nerveux. Les différentes structures corticales antérieures et postérieures impliquées dans la création des représentations associées aux émotions à valence positive/négative et dans la prise de décision sont toujours en développement entre sept et dix ans [9,17,33].

Le ressenti émotionnel positif facilite la prise de décision chez l'adulte sain [18]. Son intégration et son expression sont altérées par l'existence d'une pathologie neurodéveloppementale. Il a été révélé, par exemple, que des enfants autistes de trois ans présentent une défaillance dans la réponse à la présentation d'un visage évoquant la peur : ils ne feront pas de distinction entre les stimuli de peur et ceux qui sont neutres, montrant ainsi qu'ils se trouvent dans l'incapacité de ressentir et symboliser les émotions exprimées, contrairement à des enfants tout-venant du même âge [13]. Dans le même esprit, les études neuropsychologiques réalisées chez des enfants porteurs des lésions frontales et préfrontales mettent en évidence des dysfonctionnements de la sphère émotionnelle et de la prise de décision [23]. Des enfants souffrant des lésions préfrontales présentaient un déficit du ressenti de l'embarras, de la honte ou encore de la sympathie, étaient également dans l'incapacité d'apprendre par leurs erreurs, d'en envisager les conséquences, et de prendre des décisions en faisant des choix [12]. Des grandes difficultés dans le ressenti des émotions s'exprimant par un manque d'empathie et un comportement impulsif ainsi qu'un mode de prise de décisions très défaillant suite à une lésion frontale gauche survenue à l'âge de sept ans ont également été rapportés [15].

Les études précédentes montrent qu'une pathologie neurodéveloppementale ou neuropsychologique modifie le ressenti et l'expression de l'émotion ainsi que la prise de décision chez l'enfant. Mais qu'en est-il du ressenti émotionnel positif chez l'enfant présentant une pathologie psychiatrique classée dans les autres troubles envahissants du développement comme la dysharmonie psychotique ? Cette question n'a jamais fait l'objet d'une étude particulière, à notre connaissance. Toutefois, l'étude du ressenti positif chez ces enfants présente un intérêt particulier du fait de la richesse de son tableau clinique. 
Décrite dans la Classification française des Troubles mentaux de l'Enfant et de l'Adolescent [27] et inscrite dans les autres troubles de l'envahissement du développement dans la Classification internationale des Maladies [11], la dysharmonie psychotique se caractérise par la coexistence des perturbations de la sphère affective (anxiété, débordements émotionnels, grande excitation), et des difficultés dans la sphère cognitive. Il s'agit d'un fonctionnement psychique dominé par des mécanismes psychotiques, préservant cependant les capacités d'adaptation à la réalité et de contrôle des irruptions fantasmatiques et des affects [25]. Sa symptomatologie se caractérise par une grande excitation, de l'agressivité, la mise en place de processus autocalmants, voire une rupture avec le réel.

L'objectif de notre étude est double. Il s'agit dans un premier temps de tester si les enfants présentant une dysharmonie psychotique ressentent au même titre que les enfants «tout-venant»des émotions positives telles que l'«amour», la «joie» et la «fierté » définies par Lazarus [20] et, dans un second temps, de déterminer si le ressenti des émotions positives est lié à la prise de décision chez l'enfant présentant une dysharmonie psychotique. Dans l'affirmative, nous nous attendons à ce qu'une corrélation existe entre les deux variables chez les deux groupes d'enfants étudiés. Deux groupes d'enfants, l'un clinique, composé d'enfants présentant une dysharmonie psychotique, et l'autre, contrôle, composé d'enfants «tout venant » tous âgés de huit ans ont été constitués. Les enfants ont été invités, dans un premier temps, à écouter des histoires susceptibles de leur procurer un ressenti émotionnel positif et à choisir la fin de chaque histoire, et dans un second temps, à estimer l'intensité de l'émotion ressentie sur une échelle d'évaluation.

\section{Matériel et Méthode}




\subsection{Population}

Un groupe clinique composé d'enfants présentant une «dysharmonie psychotique » et un autre groupe contrôle composé d'enfants « tout venant » constituent la population.

Le groupe clinique comprend onze enfants, dont neuf garçons et deux filles. Leur âge moyen est de huit ans et deux mois (écart-type = un an et deux mois). Ils sont suivis en hôpital de jour. Afin de constituer un groupe homogène, tous présentent les signes du tableau psychiatrique de «dysharmonie psychotique »[11,27]. Ils ont été diagnostiqués par un psychiatre expérimenté de l'hôpital.

Leur niveau d'acquisition scolaire varie d'un niveau de maternelle à un niveau de début de CE2. Ils sont scolarisés :

- à mi-temps dans une école classique sans Assistance de Vie scolaire : deux enfants ;

- à mi-temps dans une école classique avec Assistance de Vie scolaire : deux enfants ;

- à mi-temps en Classe d'Intégration scolaire : un enfant ;

- à l'école spécialisée de l'Institut psychiatrique : six enfants.

Leur niveau d'adaptation est moyen. Leur verbalisation ne présente pas de difficultés notables. Aucun des enfants du groupe clinique n'a de problèmes de compréhension verbale. Le groupe clinique est apparié en âge et en sexe avec le groupe d'enfants « tout venant».

Le groupe d'enfant « tout venant » comprend onze enfants, dont huit garçons et trois filles. Leur âge moyen est de huit ans et trois mois (écart-type $=$ un an et un mois). Il est constitué d'enfants scolarisés classiquement à l'école primaire. Aucun n'a de pathologie psychiatrique avérée. Les enfants sont naïfs par rapport aux objectifs de l'étude. Les autorisations nécessaires pour la réalisation de cette étude ont été demandées et accordées par leurs familles.

\subsection{Procédure}

\subsubsection{Histoires}

Un jeu, consistant pour l'enfant à écouter trois histoires lues par l'expérimentateur et à en choisir la fin, a été créé. Chaque histoire est illustrée par trois dessins successifs, chacun correspondant à un moment précis de la narration et ils sont présentés sur l'écran d'un 
ordinateur. Tous les dessins défilent à un rythme constant choisi au préalable. Après une préétude visant à identifier, parmi les émotions positives les plus étudiées dans la littérature, celles qui sont les plus adaptées pour les âges sélectionnés, trois scénarios ont été élaborés : un premier relatif à l'《amour», un deuxième intéressant la «joie », et un troisième concernant la «fierté ». Les émotions étudiées correspondent en réalité à la classification d'émotions positives fournie par Lazarus [20] et ont pour but de susciter un état émotionnel positif chez l'enfant. Les illustrations et les récits ont été librement inspirés d'ouvrages pour enfants [14,19]. Le personnage (féminin ou masculin), similaire d'une histoire à l'autre, vit une émotion positive (figure 1). Des termes à connotation positive (par exemple «Il fait beau », «Joli », etc.) sont utilisés, ou non, selon le scénario. L'ordre de passation de ces trois scénarios est tiré au hasard pour chaque sujet.

\section{Insérer la figure 1 ici}

À la fin du récit de chaque histoire, l'enfant est invité à prendre une décision à l'aide de boutons réponses (rouge ou bleu) du clavier de l'ordinateur. Sa tâche est de sélectionner entre deux images, représentant simultanément deux fins possibles, celle qu'il «trouve le mieux pour finir l'histoire ». Les deux images sont situées côte à côte sur l'écran, et encadrées de couleur rouge (à gauche) ou bleue (à droite). Bien que le contenu de chaque histoire soit différent, les situations finales sont similaires d'une émotion à l'autre.

Pour les deux groupes, l'expérimentateur explique aux enfants qu'il va leur raconter des histoires et qu'il faudrait qu'ils écoutent bien. Il est également expliqué aux enfants qu'ils verront sur l'écran les images qui correspondent à chaque histoire. Il leur est demandé de choisir en appuyant sur un bouton réponse (rouge ou bleu) la fin de chaque histoire.

Nous enregistrons, pour chaque scénario, le temps de prise de décision. Ce temps est défini comme étant la durée qui s'écoule entre l'apparition sur l'écran des deux fins proposées à l'histoire et le moment où l'enfant donne sa réponse par appui sur le bouton réponse. Il est automatiquement enregistré par l'ordinateur.

\subsubsection{L'autoévaluation}

Immédiatement après chaque histoire, l'enfant est invité à montrer, le long d'une flèche croissante, lequel parmi cinq visages [28], du plus neutre au plus souriant, correspond à l'émotion qu'il a ressentie. L'utilisation d'images représentant différents degrés d'états 
émotionnels positifs, en nuançant principalement le sourire du personnage, permet à l'enfant de préciser son ressenti, sans avoir recours à l'utilisation du langage. L'expérimentateur demande à l'enfant de montrer sur la flèche comment il serait content s'il était lui-même « dans l'histoire ». S'il n'aurait rien ressenti de spécial (1), ou est ce qu'il aurait été un peu content (2), content (3), très content (4), ou énormément content (5). Le degré du ressenti émotionnel subjectif correspond à l'intensité de l'émotion suscitée par le scénario, et est donc coté de 1 à 5 (intensité la plus élevée).

\section{Résultats}

Les résultats vont être présentés en deux sessions. La première concerne les distributions du temps de prise de décision et du ressenti émotionnel ; la deuxième la relation entre les temps de prise de décision et l'évaluation du ressenti émotionnel. Ces deux sessions vont être développées pour le groupe d'enfants présentant une dysharmonie psychotique et pour le groupe d'enfants « tout venant».

La mise en relation entre temps de prise de décision et ressenti émotionnel peut être potentiellement réalisée au moyen de divers indices de corrélation. Le choix d'un indice particulier est cependant dépendant de la forme des distributions des variables.

\subsection{Les distributions du temps de prise de décision et du ressenti émotionnel}

Les distributions de temps de prise de décision sont symétriques et s'apparentent à une courbe de Gauss, et ce quels que soient le groupe et l'émotion étudiés. Ces distributions suivent donc la loi normale.

Les distributions de l'intensité du ressenti émotionnel pour les trois modalités choisies (amour, fierté et joie) sont asymétriques, ne suivant pas la loi normale quel que soit le groupe.

Les intensités du ressenti émotionnel ne diffèrent pas entre le groupe clinique et le groupe contrôle, tant en ce qui concerne l' « amour » (T de Wilcoxon $=15, \mathrm{n}=8, \mathrm{p}>0,05)$, que la «fierté » $(\mathrm{T}$ de Wilcoxon $=10, \mathrm{n}=7, \mathrm{p}>0,05)$, et la «joie » $(\mathrm{T}$ de Wilcoxon $=6$, $\mathrm{n}=6, \mathrm{p}>0,05)$. 


\subsection{Relations entre le temps de prise de décision et l'intensité du ressenti}

Étant donné les formes des temps de prise de décision et des distributions des ressentis des émotions, la mise en relation des deux variables a été réalisée au moyen d'un test de corrélation non paramétrique (coefficient de corrélation par rangs de Spearman).

\subsubsection{Groupe d'enfants présentant une dysharmonie psychotique}

L'analyse statistique indique que les temps de prise de décision pour les émotions «amour » et «fierté » sont positivement corrélés avec l'intensité du ressenti de l'émotion ( $\rho$ de Spearman $=0,737 \mathrm{p}<0,01$ unilatéral pour l' «amour $» ; \rho$ de Spearman $=0,618$ $\mathrm{p}<0,05$ unilatéral pour la «fierté »). En revanche, les deux variables ne sont pas corrélées pour la troisième émotion étudiée ( $«$ joie $»[\rho$ de Spearman $=0,476 \mathrm{p}>0,05])$.

\subsubsection{Groupe d'enfants « tout venant»}

L'analyse statistique indique que les temps de prise de décision pour les trois émotions étudiées sont positivement corrélés avec l'intensité du ressenti de l'émotion («amour »[ $\rho$ de Spearman $=0,616 \mathrm{p}<0,025$ unilatéral $]$; «fierté » $[\rho$ de Spearman $=0,644 \mathrm{p}<0,05$ unilatéral $]$; «joie » $[\rho$ de Spearman $=0,562 \mathrm{p}<0,025$ unilatéral $]$ ).

Dans les figures 2 et 3, les graphiques mettent en relation, pour chaque sujet, son rang de temps de prise de décision et son rang d'intensité de ressenti.

\section{Insérer les figures 2 et 3 ici}

\section{Discussion}

Notre recherche visait à tester si les enfants présentant un trouble envahissant du développement, tel qu'une dysharmonie psychotique, pourraient, au même titre que les enfants tout-venant, ressentir les émotions positives, et s'il existait une relation entre le temps de prise de décision et le ressenti émotionnel positif. Dans l'hypothèse où la prise de décision serait, au moins en partie, liée à l'intensité du ressenti émotionnel, nous nous attendions à ce qu'une corrélation existe entre les deux variables chez les deux groupes d'enfants étudiés. 
Notre recherche aboutit à deux types de résultats. D'un côté, l'intensité du ressenti émotionnel ne diffère pas entre les deux groupes d'enfants. De l'autre, il y a une corrélation positive entre ces deux variables pour les deux groupes d'enfants étudiés, à l'exception du ressenti de la « joie » pour le groupe d'enfants présentant une dysharmonie psychotique.

Ainsi, les données obtenues sont compatibles avec des résultats selon lesquels les enfants âgés entre trois et dix ans sont non seulement capables de discriminer les émotions à valence positive [16,31] et d'accorder à une expression corporelle ou visuelle donnée l'émotion correspondante, mais aussi d'attribuer à autrui des états mentaux [3,4]. Néanmoins, nos résultats vont au-delà de ces travaux en ce qu'ils montrent pour la première fois expérimentalement, à notre connaissance, que les enfants présentant une dysharmonie psychotique ressentent, au même titre que les enfants tout-venant, les émotions positives telles que la «joie », l'«amour» et la «fierté », émotions fournies par Lazarus [20]. En d'autres termes, ces résultats révèlent que tous les enfants examinés sont capables d'empathie. En tant que tels, ils sont en faveur de la théorie selon laquelle les enfants présentant une dysharmonie psychotique auraient conservé les capacités d'adaptation à la réalité et des affects [25].

Notre recherche montre aussi que plus l'intensité du ressenti émotionnel est élevée, plus le temps de prise de décision est long. Cette corrélation est avérée pour les trois émotions - «amour », «fierté », «joie » - chez les enfants du groupe contrôle et seulement pour l'« amour » et la «fierté » chez les enfants du groupe clinique.

En essayant de comprendre les composants de cette interdépendance, nous esquisserons les raisons pour lesquelles le ressenti de la «joie » n'est pas corrélé avec le temps de prise de décision chez les enfants présentant une dysharmonie psychotique. Dans notre étude, les enfants ont d'abord été invités à écouter attentivement une histoire et à regarder des scènes visuelles représentant certains moments de l'histoire lue, simultanément, leur permettant de ressentir la «joie», la «fierté » et l'«amour». Les enfants devaient décider quelle fin accorder à chaque histoire en faisant un choix parmi deux possibles. Le temps de prise de décision mesuré correspond à la durée qui s'écoule entre la fin de récit de l'histoire et l'appui sur le bouton-réponse. Cette durée dépendrait du temps total de traitement de l'émotion, le dernier impliquant au moins trois niveaux : sensoriel, perceptif, et conceptuel. Le niveau de base est «sensoriel » et qualifie les précâblages existant entre les stimuli visuels et auditifs. Les enfants se trouvent dans une situation de transfert intermodal auditif et visuel. Ils écoutent des histoires qui sont, tout au moins en partie, illustrées et présentées sur l'écran de l'ordinateur. Le deuxième niveau, d'ordre « perceptuel», consiste à traiter les informations auditives et visuelles présentées pour actualiser les schèmes correspondants mémorisés. Le 
troisième niveau, d'ordre «conceptuel », permet de rappeler des représentations mentales associées aux expériences émotionnelles intériorisées. En fait, il ne s'agit pas d'une simple organisation séquentielle et hiérarchique ascendante puisque des mécanismes de rétroaction neuronaux permettent une influence des représentations mentales sur les autres niveaux. Une fois le traitement réalisé, l'enfant prend sa décision en effectuant un choix. La charge mentale nécessaire est élevée et dépendrait de la nature de la tâche demandée, d'autant plus qu'à la fin de l'histoire, certains enfants se sont amusés, comme ils ont déclaré, à « inventer une autre fin ». Soit les situations de fin d'histoire proposées n'étaient pas familières pour l'enfant, soit les fins présentées n'étaient pas satisfaisantes ou ne correspondaient pas aux plans d'actions possibles connues ou imaginés par l'enfant. En d'autres termes, les séquences émotionnelles proposées ne pouvaient être intégrées à des schèmes déjà existants, ce qui retarderait le temps de prise de décision par augmentation de la charge mentale.

Dans ce contexte, l'absence de corrélation entre le ressenti de la « joie » et la prise de décision dans le groupe clinique ne peut être attribuée ni à une difficulté de compréhension des histoires permettant le ressenti émotionnel et de la procédure utilisée, ni à la qualité de réactivité de prise de décision, différents pré-tests ayant permis de sélectionner les émotions à étudier et d'adapter l'étude aux enfants. Elle peut en revanche être comprise comme étant l'expression manifeste de la difficulté d'intégration des différentes étapes du traitement de l'émotion qui permettent la prise de décision. Elle serait probablement due à l'impossibilité d'accéder aux étapes «perceptuelle » et «conceptuelle», comportement qui témoignerait de la difficulté de faire émerger des représentations internes associées au vécu de cette émotion. Vont dans le sens de cette hypothèse des observations cliniques qui relatent que chez certains enfants dysharmoniques le vécu de plaisir, la joie par exemple, peut parfois provoquer une tension pulsionnelle intense qui envahirait tout le champ de la conscience jusqu'à contraindre de mener des actions [25]. En d'autres termes, ce résultat révèle que la pathologie étudiée enraye les processus neurocognitifs de l'émotion.

Si, chez l'adulte, l'émotion positive permet de cibler les éléments importants et facilite la prise de décision [18], nos résultats montrent que chez l'enfant, et dans certaines circonstances, le ressenti de l'émotion positive entrave la prise de décision. En fait, la prise de décision de choix nécessite de sélectionner une réponse et d'en inhiber d'autres. Étant donné que les structures neurales antérieures et postérieures impliquées dans la création des représentations émotionnelles et la prise de décision sont toujours en développement à huit ans $[9,17,33]$, il est possible que le processus de sélection de la réponse soit ralenti, voire inhibé, du fait de l'immaturité des capacités d'autorégulation. Cela prend une signification 
particulière quand il s'agit d'enfants présentant un trouble envahissant du développement comme la dysharmonie psychotique. Que cela relève de l'immaturité ou de la pathologie ou des deux à la fois, et bien que la notion de «psychose » chez l'enfant ne soit pas consensuelle, notre recherche démontre pour la première fois expérimentalement, à notre connaissance, que les enfants présentant une dysharmonie psychotique ressentent les émotions telles que l'«amour », la «joie » et la «fierté », de la même manière que les enfants « tout venant ». Elle révèle également que le ressenti positif, tel que la «joie », enrayerait l'émergence des représentations associées au vécu de cette émotion du fait d'une forte tension pulsionnelle chez l'enfant présentant une dysharmonie psychotique. Des travaux supplémentaires sont sans doute nécessaires pour étayer davantage cette question, mais notre travail montre que le ressenti de certaines émotions positives peut freiner la prise de décision chez l'enfant présentant ou non une dysharmonie psychotique.

\section{Conflit d'intérêt : à compléter par l'auteur}

\section{Références}

[1] Barbas H. Anatomic organization of basoventral and mediodorsal visual recipient prefrontal regions in the rhesus monkey. J Comp Neurol 1988;276:313-42.

[2] Barbas H. Organization of cortical afferent input to the orbitofrontal area in the rhesus monkey. Neuroscience 1993;56:841-64.

[3] Baron-Cohen S, Leslie AM, Frith U. Does the autistic child have a theory of mind? Cogn $1985 ; 21: 37-46$.

[4] Baron-Cohen S, Ring H. A model of the mind-reading system: Neuropsychological and neurobiological perspectives. In: Mitchell P, Lewis C. Origins of an understanding of mind. Hillsdale, NJ: Erlbaum; 1994. p. 183-207.

[5] Berthoz A. La décision. Paris: Odile Jacob; 2003.

[6] Bowlby J. Attachement et perte : l'attachement. Paris: PUF « Le fil rouge »; 1978.

[7] Carmichael ST, Price JL. Architectonic subdivision of the orbital and medial prefrontal cortex in the macaque monkey. J Comp Neurol 1994;346:366-402.

[8] Carr L, Iacoboni M, et al. Neural mechanisms of empathy in humans: A relay from neural systems for imitation to limbic areas. PNAS 2003;100:5497-502.

[9] Casey BJ, Tottenham N, et al. Imaging the developing brain: What have we learned about cognitive development? Tr Cogn Sci 2005;9:104-10. 
[10] Clark L, Manes F. Social and emotionnal decision-making following frontal lobe injury. Neurocase 2004;10:398-403.

[11] Classification internationale des maladies : CIM, octobre 2007.

[12] Damasio AR. Spinoza avait raison : joie et tristesse, le cerveau des émotions. Paris: Odile Jacob; 2003.

[13] Dawson G, Webb SJ, et al. Young children with autism show atypical brain responses to fearful versus neutral facial expressions of emotion. Dev Sci 2004;7:340-49.

[14] Elzbieta La nuit de l'Etoile d'or. Paris: Pastel; 1993.

[15] Eslinger PJ, Grattan, LM, et al. Developmental consequences of childhood frontal lobe damage. Arch neurol 1992;49:764-69.

[16] Garon N, Moore C. Complex decision-making in early childhood. Brain Cogn 2004;55:158-70.

[17] Gogtay N, Giedd JN, et al. Dynamic mapping of human cortical development during childhood through early adulthood. Proc Nat Acad Sci USA 2004;101:8174-9.

[18] Isen AM, Rosenzweig AS, et al. The influence of positive affect on clinical problem solving. Med dec mak 1991;11:221-7.

[19] Labbé B, Puech M, et al. Les goûters philo. La compil T1. Milan Edition. Paris: Milan Editions; 2002.

[20] Lazarus R. Emotion and adaptation. Oxford: Oxford University Press; 1991.

[21] LeDoux JE. Emotion circuits in the brain. An Rev Neurosci 2000;23:155-84.

[22] Leslie KR, Johnson-Frey SH, et al. Functional imaging of face and hand imitation: Toward a motor theory of empathy. Neuroimage 2004;21:601-7.

[23] Lussier F, Flessas J. Syndrômes frontaux. In: Lussier F, Flessas J. Neuropsychologie de l'enfant : troubles développementaux et de l'apprentissage. Paris: Dunod; 2001. P. 309-58.

[24] Main M. Introduction to the special section on attachment and psychopathology: 2. Overview of the field of attachment. J Cons Clin Psychol 1996;64:237-43.

[25] Marcelli D. Enfance et psychopathologie. Paris: Masson; 1982.

[26] Messinger DS. A measure of early joy? In: Ekman P, Rosenberg EK, What the face reveals: Basic and applied studies of spontaneous expression union the Facial Action Coding System (FACS). Oxford: Oxford University Press; 2005. p. 350-3.

[27] Misès R, Quemada N, Botbol M, Bursztejn C, Durand B, Garrabé J, et al. Classification française des troubles mentaux de l'enfant et de l'adolescent : CFTMEA R-2000. (en ligne) http://psydoc-fr.broca.inserm.fr:16080/biblo.bd/cftmea/ 
[28] Monfort M, Monfort-Juarez I. L'Esprit des Autres: Un support visuel pour l'entraînement des habiletés pragmatiques chez l'enfant. Madrid: Entha Ediciones; 2001.

[29] Morecraft RJ, Geula C, et al. Cytoarchitecture and neural afferents of orbitofrontal cortex in the brain of the monkey. J Comp Neurol 1992;323:341-58.

[30] Rolls ET. The functions of the Orbitofrontal cortex. Brain Cogn 2004;55:11-29.

[31] Russell JA. Culture, scripts, and children's understanding of emotion. In: Saarni C, Harris PL. Children's understanding of emotion. Cambridge; Cambridge University Press: 1989. p. 293-313.

[32] Siegel DJ. The developing mind: How the relationships and the brain interact to shape who we are. New York, London: Guilford Press; 1999.

[33] Sowell ER, Thompson PM, et al. Longitudinal Mapping of Cortical Thickness and Brain Growth in Normal Children. J Neurosci 2004;24:8223-31.

\section{Figures}

Figure 1. Exemple d'illustration d'une partie du scénario « la joie »

Figure 2. Enfants «tout venant »: temps de prise de décision et intensité du ressenti

Figure 3. Enfants présentant une dysharmonie psychotique: temps de prise de décision et intensité du ressenti 


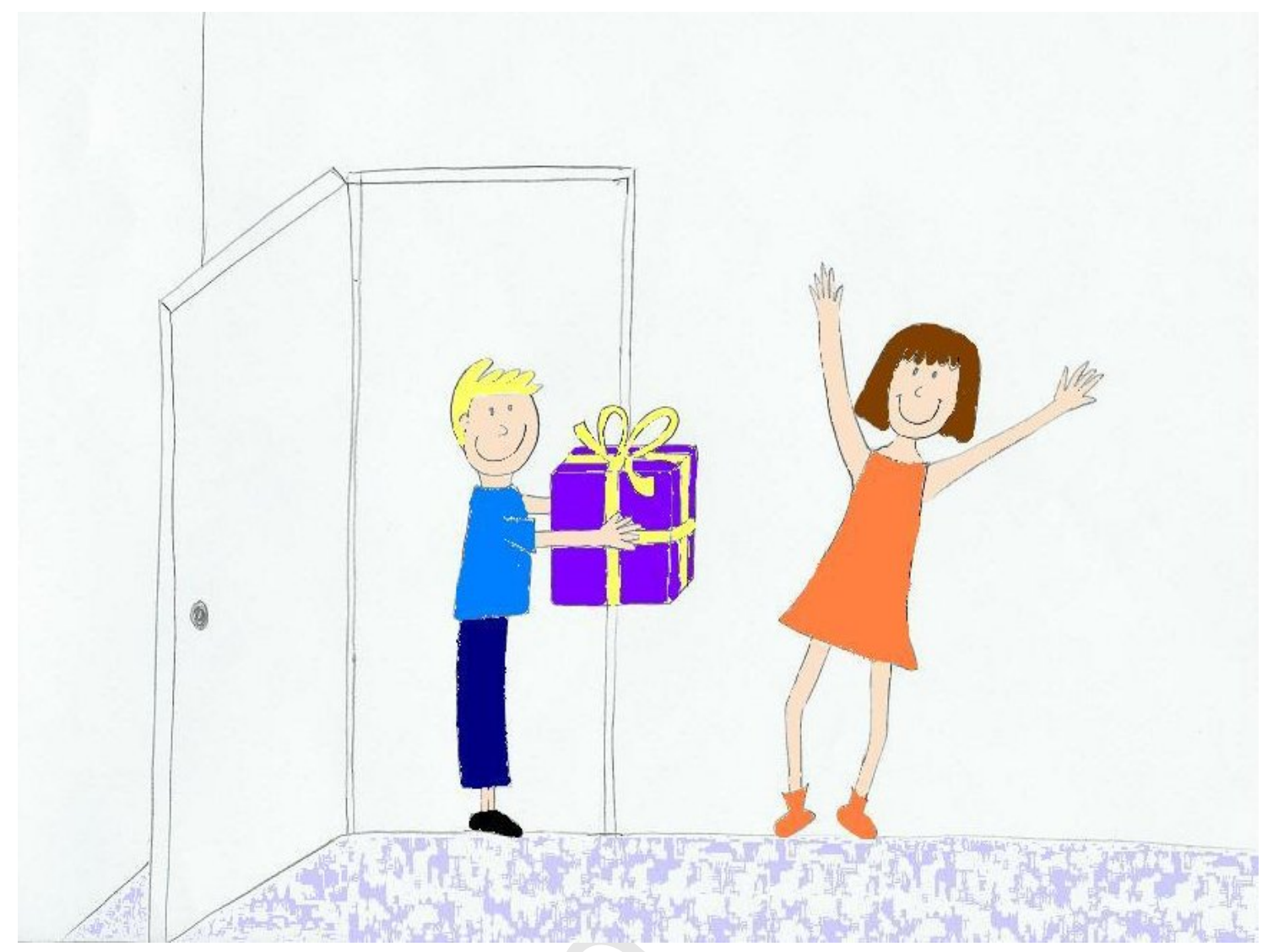

Figure 1. Exemple d'une partie du scénario « joie » :

le personnage vit une émotion positive 


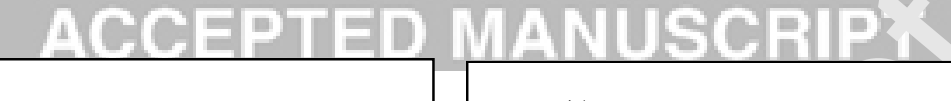
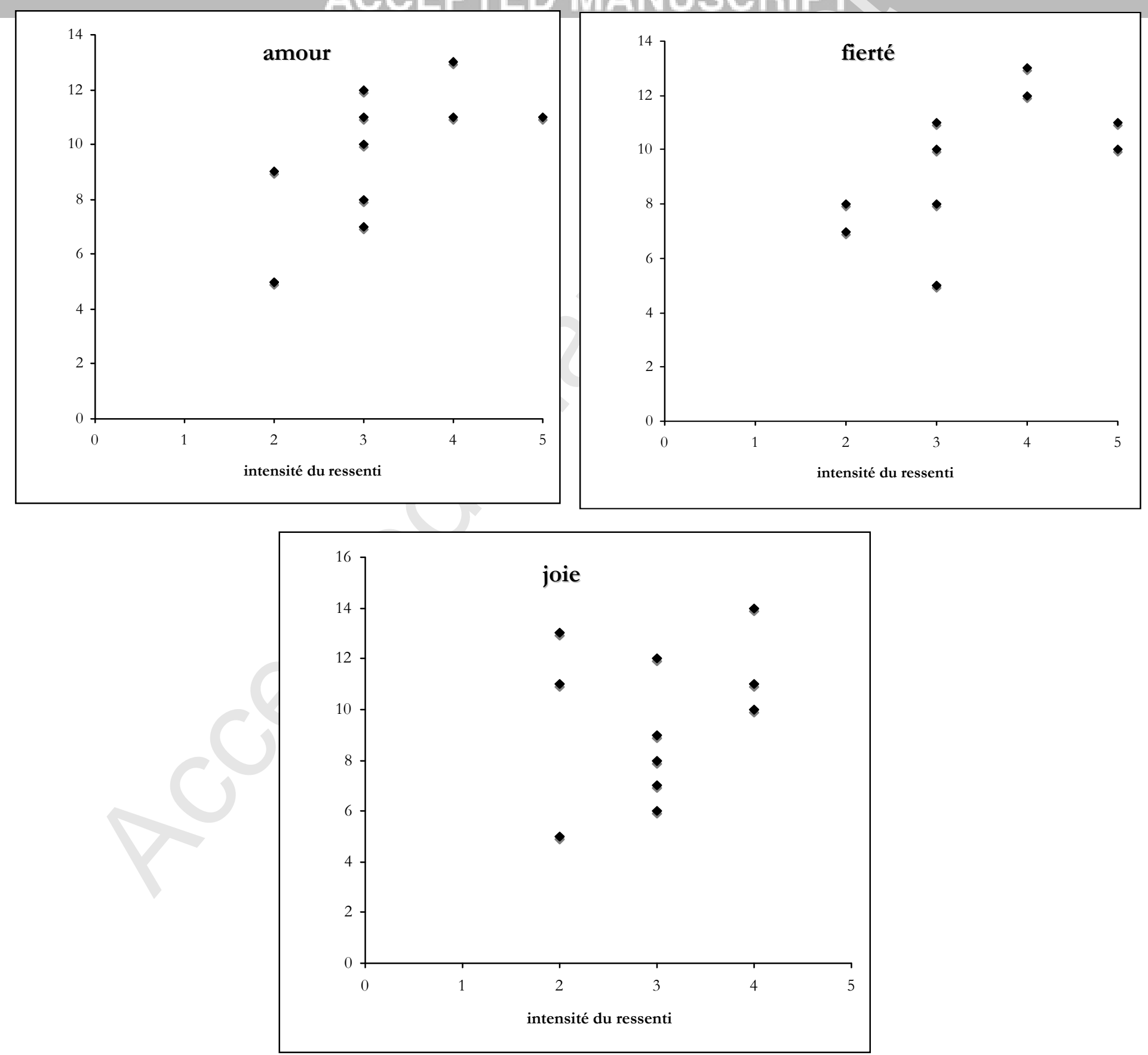

Figure 2. Enfants «tout venant» : corrélation entre le ressenti émotionnel et le temps de prise de décision 
Page 19 of 19 